\title{
Boosting Phonological Fluency Following Leftward Prismatic Adaptation: A New Neuromodulation Protocol for Neurological Deficits?
}

Patrizia Turriziani

University of Palermo

Gabriele Chiaramonte

University of Palermo

Giuseppa Renata Mangano

University of Palermo

Rosario Emanuele Bonaventura

University of Palermo

Daniela Smirni

University of Palermo

Massimiliano Oliveri ( $\square$ Massimiliano.oliveri@unipa.it)

University of Palermo

\section{Research Article}

Keywords: Prism adaptation (PA), phonological, neurological

DOI: https://doi.org/10.21203/rs.3.rs-109260/v1

License: (1) This work is licensed under a Creative Commons Attribution 4.0 International License.

Read Full License 


\section{Abstract}

Prism adaptation (PA) has been recently shown to modulate a brain frontal-parieto-temporal network, with an increase of excitation of this network in the hemisphere ipsilateral to the side of prismatic deviation. This effect raises the hypothesis that left prismatic adaptation, modulating the excitability of frontal areas of the left hemisphere, could modulate subjects' performance on linguistic tasks that map on those areas.

To test this hypothesis, sixty-one healthy subjects participated in experiments in which leftward, rightward or no-PA were applied before the execution of a phonological fluency task, i.e. a task with strict left hemispheric lateralization and mapping onto frontal areas.

Leftward-PA significantly increased the number of words produced compared with the pre-PA $(p=.0017)$, R-PA $(p=.00013)$ and no-PA ( $p=.0005)$ sessions. In contrast, rightward-PA did not significantly modulate phonological fluency compared with the pre-PA $(p=.92)$ and no-PA $(p=.99)$ sessions.

The effect of leftward PA on phonological fluency correlated with the magnitude of spatial aftereffect, i.e. the spatial bias towards the side of space opposite to prismatic deviation following prisms removal $(r=$ $.51 ; p=.04)$.

The present findings document for the first time modulation of a language ability following prismatic adaptation. The results could have a huge clinical impact in neurological populations, opening new strategies of intervention for language and executive dysfunctions.

\section{Introduction}

Prism adaptation (PA) is a form of visuomotor adaptation to displaced vision (for review see ${ }^{1}$ ) and it has been shown to modulate a wide range of behaviors (for review see ${ }^{2}$ ) in addition to the well-known application in patients with right hemispheric lesion and spatial neglect (for a review ${ }^{3}$ ).

The majority of observations indicate that prism adaptation acts both on space representation and on other features interacting with space representation. For example, in healthy subjects leftward PA induces a sort of left minineglect, counteracting the physiological leftward bias called pseudoneglect ${ }^{4,5}$. PA aftereffects have also been reported in visual search $^{6}$, endogenous and/or exogenous orienting of attention ${ }^{7}$, spatial/temporal representation $8,910,11,12,13,14$, visually guided actions ${ }^{15}$, auditory representation ${ }^{16}$, chronic pain ${ }^{17}$, constructional disorders ${ }^{18}$ and reward-based learning ${ }^{19}$.

Recent research suggested that visuomotor adaptation elicited by PA can also induce modulation of frontal areas ipsilateral to prismatic deviation, i.e. contralateral to the after-effect induced. Magnani et al. ${ }^{11}$, in a study using paired-transcranial magnetic stimulation (TMS) in healthy subjects, first reported modulation of excitatory brain circuits on the motor cortex specific to the direction of the visual shift induced by prismatic lenses: left deviation increased excitation of the left motor cortex, while right 
deviation increased excitation of the right motor cortex, as tested with the amplitude of motor evoked potentials.

Bracco et al. ${ }^{20}$ reproduced these findings in a study combining TMS, transcranial direct current stimulation and PA in healthy subjects. Prismatic adaptation increased excitability of the motor cortex ipsilateral to the deviation, as tested with TMS, in a manner similar as anodal tDCS did. The combination of the two excitatory interventions (i.e. PA and anodal tDCS) induced homeostatic plasticity effects, reducing motor cortical excitability. The same research group ${ }^{21}$ showed that prismatic deviation induces an increase of the power of beta oscillations in the frontal areas of the hemisphere ipsilateral to the optical deviation during motor preparation but not visual attention tasks.

These findings suggest that prismatic adaptation can neuromodulate brain excitability of a brain network ipsilateral to the deviation, with effects that could impact the cognitive functions subserved by that network. This view suggests that left PA, modulating the excitability of frontal areas of the left hemisphere, could modulate subjects' performance on linguistic tasks that map on those areas.

In the present study, we tested this assumption by investigating the power of PA in modulating phonemic fluency tasks. We chose to investigate phonological fluency because it shows a strong left hemispheric lateralization in frontal areas ${ }^{22}$ and it has been studied with other neuromodulatory techniques ${ }^{23}$.

Phonological fluency tasks require search, access, selection, retrieval and pronunciation of as many words as possible in a restricted time, based on a predefined criterion of a target letter. Therefore, fluency tasks are included in many neuropsychological batteries in that they probe cognitive functions at the interface between language and executive processing. As such, phonological fluency can be impaired in a variety of clinical populations, including aphasia and dementia $24,25,26$.

We assumed that adaptation to a leftward optical deviation should increase subjects' performance compared to both rightward optical deviation and no adaptation conditions.

\section{Methods}

\section{Subjects}

Sixty-one healthy subjects (10 males, mean age: $23.1 \pm 2.4$ years) volunteered to participate in this experiment. All participants were native Italian speakers, right-handed, had a normal or corrected-tonormal vision and reported no history of neurological or psychiatric disease.

Thirty-one subjects were randomly allocated in the experimental group (4 males, mean age: $23.48 \pm 2.32$ years). Participants were assigned to a leftward Prismatic

Adaptation group (L-PA; $\mathrm{n}=16$; mean age $=23.43 \pm 1.86$ years) or a rightward Prismatic Adaptation group $\left(\mathrm{R}-\mathrm{PA} ; \mathrm{n}=15 ;\right.$ mean age $=23.53 \pm 2.79$ years). The l-PA group wore $20^{\circ}$ left shifting prismatic 
lenses and the r-PA group wore $20^{\circ}$ right shifting prismatic lenses. Participants handedness was assessed using the Edinburgh Handedness Inventory ${ }^{74}$.

In the control group, there were 30 right-handed healthy participants ( 4 males, mean age $=24.8 \pm 2.34$ years).

All subjects gave written informed consent for participation in the study, that was approved by the ethical committee of the University of Palermo (approval n. 25/2020). The experiments were done in accord to the principles of Declaration of Helsinki.

\section{Neuropsychological assessment}

The experimental group underwent a neuropsychological evaluation. Digit Span forward and backward ${ }^{75}$, Symbol Digit Modalities Test ${ }^{76}$ (SDMT), Modified Five Point Test ${ }^{77}$ (MFPT); a short version of the Stroop Colour-Word Test $^{78}$, Raven's Advanced Progressive Matrices ${ }^{79}$.

\section{Prismatic adaptation procedure}

The procedure for prismatic adaptation was similar to that adopted in previous studies ${ }^{9,12,13 \text {, }}$

For PA, subjects sat in front of a box (height $=30 \mathrm{~cm}$, depth $=34 \mathrm{~cm}$ at the center and $18 \mathrm{~cm}$ at the periphery, width $=72 \mathrm{~cm}$ ) open on two sides: the side facing the subjects and the opposite side, facing the experimenter. The experimenter placed a pen as a visual target at the distal edge of the top surface of the box, in one of three randomly determined positions: a central position $\left(0^{\circ}\right), 21^{\circ}$ to the left of the center, and $21^{\circ}$ to the right of the center. Subjects were asked to keep their right hand at the level of the sternum and then to point toward the visual target using the right index finger; the experimenter recorded the end position of the subject's pointing direction. The pointing task was performed in four experimental conditions: pre-exposure, exposure (early-exposure, late-exposure) and post-exposure.

In the early-exposure (first 9 trials while wearing prisms), late-exposure (last 9 trials while wearing prisms), and exposure conditions, the subjects performed the task with prismatic lenses inducing a rightward or leftward $20^{\circ}$ shift. The pointing procedure was visible, i.e. the subjects could see the trajectory of the arm movement.

In the post-exposure condition, performed immediately after prisms removal, the subjects were required to look at the target and to make their pointing movements with their eyes closed as in the pre-exposure condition. Thus, in this condition the trajectory of the arm movement was invisible for the subject.

Exposure condition comprehended 90 trials, while each of the other conditions comprehended 30 trials. All the pointing trials were equally and randomly distributed toward the three marked positions of the panel.

Phonemic fluency tasks 
Two phonemic fluency tasks, standardized for the Italian population, were used ${ }^{8081}$. Both tasks require participants to generate as many words as possible starting with a given letter within 1 min, excluding proper nouns and words differing only for the suffix. In one of the two phonemic fluency tasks, the 3 letters used were " $F$ " " $A$ " " $S$ ". In the second task, the 3 letters used were " $F$ " " $P$ " " $L$ ".

\section{Experimental procedure}

Both the L-PA and the R-PA groups and the control group participated in two testing sessions over two separate days, with an interval of seven days between sessions (Fig. 1).

In the first testing session, the two experimental groups were given the cognitive baseline tasks and the phonemic fluency task (FAS or FPL).

In the second testing session, the two experimental groups were first administered the PA procedure (L-PA or R-PA), immediately followed by one of the two phonemic fluency tasks.

The control group was administered one of the two phonemic fluency tasks (FAS or FPL) in the first testing session. In the second testing session, the control group was administered the other fluency task. The order of administration of the two phonemic fluency tasks was counterbalanced across the control group and randomly assigned.

\section{Statistical analysis}

\section{Prismatic adaptation}

Error reduction. To verify whether subjects adapted to prismatic deviation, showing an error reduction following rightward or leftward deviation, we compared their displacement measure in the pre-exposure (visible pointing) condition with that of the first three (early- exposure condition) and the last three trials (late-exposure condition) of the exposure Condition (more details on this procedure can be found $\mathrm{in}^{82}$ ). A difference between a pre-exposure condition and the early-exposure condition is expected due to the rightward or leftward displacement induced by prism exposure. On the other hand, no difference is expected between pre-exposure and the late-exposure condition in the assumption of an almost perfect error reduction. The dependent measure in this analysis was the mean displacement (expressed as degrees of visual angle) of subjects' visible pointing. An ANOVA was conducted with Group (L-PA; R-PA) as between-groups and Condition (pre-exposure, early-exposure and late-exposure) as the within-subjects variable. Post hoc comparisons were conducted using Tukey's test.

Aftereffect. We compared the subjects' displacement in the invisible pointing in the pre-exposure and post-exposure conditions. If, after prism exposure, subjects point to the direction opposite the displacement induced by the prism, a difference is expected between the pre- and the post-exposure conditions (aftereffect). The dependent measure was the mean displacement (expressed in degrees of visual angle) of the subjects' invisible pointing responses in the pre-exposure condition and the post- 
exposure condition. An ANOVA was conducted with Group (L-PA; R-PA) as between-groups and Condition (pre-exposure, post-exposure) as a within-subjects variable. Post hoc comparisons were conducted using Tukey's test.

\section{Phonemic fluency task}

Behavioral data were analyzed with an ANOVA for repeated measures, with Condition (L-PA, R-PA, No-PA) as between-subjects factor and Session (pre-PA, post-PA) as a within-subjects factor. Post-hoc analyses were conducted with Tukey's test.

\section{Results}

Demographic and cognitive data of the experimental groups are reported in Table 1.

There were no significant differences in Age $\left(F_{1,29}=.01, p=.91\right)$, Education $\left(F_{1,29}=.0009, p=.97\right)$ and Handedness $\left(F_{1,29}=1.08, p=.30\right)$ between the two experimental groups. Similarly, there were no significant differences in the performance of the two experimental groups on the cognitive baseline tasks: Digit Span (forward $\left(F_{1,29}=.08, p=.77 ;\right.$ backward $\left.F_{1,29}=1.35 ; p=.25\right)$, SDMT $\left(F_{1,29}=.43, p=.51\right.$ ), MFPT $\left(F_{1,29}=.85 ; p=.36\right)$, Stroop test $\left(F_{1,29}=1.64 ; p=.21\right)$, RAPM $\left(F_{1,29}=.61 ; p=.44\right)$.

\section{Prismatic adaptation}

Error reduction. The ANOVA showed a significant effect of Group $\left(F_{1,29}=47.85, n p^{2}=0.20, p=.0000\right)$ and a Group $x$ Condition interaction $\left(F 2,58=38.07, n p^{2}=0.62, p=.0000\right)$. The Condition main effect was not significant $\left(F_{1,29}=1.03, n p^{2}=0.03, p=.34\right)$. The post-hoc analyses showed that, for both groups, the pointing displacement in the pre-exposure condition was significantly different from that in the earlyexposure-condition (L-PA $p=.001 ;$ R-PA $p=.006$ ).

Conversely, due to subjects' adaptation to prismatic deviation, no differences were found between preexposure and late exposure neither in the L-PA $(p=.99)$ nor the R-PA $(p=.99)$ group.

Aftereffect. The ANOVA revealed a significant effect of Group $\left(F_{1,29}=219.57, n p^{2}=0.88, p=.0000\right)$ and a Group $x$ Session interaction $\left(F_{2,58}=192.54, n p^{2}=0.86, p=.0000\right)$. The Condition main effect was not significant $\left(F_{1,29}=3.28, n p^{2}=0.10, p=.08\right)$.

The presence of after effect was confirmed by a significant difference between blind pre-exposure and blind post-exposure in both the L-PA $(p=.0001)$ and the R-PA $(p=.0001)$ groups (Fig. 2$)$.

\section{Phonemic fluency task}

The ANOVA performed on the number of words produced in the phonemic fluency tasks revealed a significant Group $x$ Session interaction $\left(F_{2,58}=7.53 ; n p^{2}=0.20, p=.001\right)$. The main effects of Group 
$\left(F_{1,29}=2.18 ; \eta p^{2}=0.07, p=.12\right)$ and Session $\left(F_{1,29}=3.87 ; \eta p^{2}=0.06, p=.06\right)$ did not reach statistical significance.

In the first Session (pre-PA), we found no significant difference between the phonemic fluency performance of the no-PA group and the two experimental groups (L-PA and R-PA). There was no significant difference between no-PA and L-PA $(p=1.00)$ or R-PA $(p=.93)$.

Interestingly, L-PA significantly increased the number of words produced compared with the pre-PA $(p=$ .0017), R-PA ( $p=.00013)$ and no-PA ( $p=.0005)$ sessions. In contrast, R-PA did not significantly modulate phonological fluency compared with the pre-PA $(p=.92)$ and no-PA $(p=.99)$ sessions (Fig. 3$)$.

In sum, we found that adaptation to a leftward optical deviation increased subjects' performance in the phonemic fluency task as compared to both rightward optical deviation and no adaptation conditions.

Additionally, we investigated whether PA affected the quality of the words (nouns or verbs) produced in the Phonemic fluency task.

The ANOVA on the number of verbs produced in the phonemic fluency tasks did not reveal any statistical significance in both the main effects of $\operatorname{Group}\left(\mathrm{F}_{1,29}=0.17 ; n p^{2}=0.68, p=.006\right)$ and Session $\left(F_{1,29}=\right.$ $\left.0.36 ; \eta p^{2}=0.54, p=.06\right)$ and Group $x$ Session interaction $\left(F_{2,58}=0.36 ; \eta p^{2}=0.54, p=.06\right)$.

Conversely, an ANOVA performed on the number of nouns produced in the phonemic fluency tasks revealed a significant Session $\left(F_{1,29}=6.42 ; \eta p^{2}=0.18, p=.01\right)$ and Group $x$ Session interaction $\left(F_{2,58}=\right.$ $\left.5.77 ; n p^{2}=0.16, p=.023\right)$. The Group $\left(F_{1,29}=3.24 ; \eta p^{2}=0.10, p=.10\right)$ did not reach statistical significance. The post-hoc tests revealed an increase of nouns after L-PA $(p=.007)$ but not after R-PA $(p=$ .99).

In sum, leftward optical deviation increases the number of nouns but not of verbs produced.

We also analyzed the mean number of syllables (number of syllables/number of words) produced in the pre-PA and post-PA during the Phonemic fluency task. The ANOVA on this variable showed a significant Group $x$ Session interaction $\left(F_{2,58}=4.71 ; n p^{2}=0.14, p=.0038\right)$. The main effects of Group $\left(F_{1,29}=0.17\right.$; $\left.\eta p^{2}=0.67, p=.006\right)$ and Session $\left(F_{1,29}=1.18 ; n p^{2}=1.18, p=.06\right)$ did not reach statistical significance. Post-hoc analyses showed that L-PA but not R-PA $(p=.57)$ increased the number of syllables $(p=.02)$.

These findings indicate that leftward PA increases not only the absolute number of words produced but also the production of words formed by a greater number of syllables.

Furthermore, we conducted Pearson correlation analyses to investigate the relationship between the effect of the PA and Phonemic fluency tasks. Differences between pre-PA and post-PA scores in Phonemic fluency tasks ( $\Delta$ phonemic fluency) were correlated with the index of prismatic adaptation (PA 
aftereffect). A positive correlation was found between $\Delta$ of phonemic fluency and the index of prismatic adaptation in the L-PA group $(r=.51 ; p=.04)$ but not in the R-PA group $(r=.46 ; p=.08)$.

\section{Discussion}

The main results of the present study show that leftward optical deviation induced by prismatic deviation is associated with improved phonemic fluency performance in healthy subjects when compared with either baseline (i.e. no optical deviation) or rightward optical deviation conditions. Improved phonemic fluency was evident either in terms of the number of words produced and in the number of syllables for each word. The increase in phonemic fluency following leftward PA was mainly evident for the grammatical category of nouns.

These results were not accounted for practice effects. Parallel forms of the task were used in baseline and post-PA sessions; moreover, the control study in the no-PA group failed to document significant increases in phonological fluency performance across repeated sessions.

Adaptation to both left and right PA induced sensorimotor aftereffects. The R-PA and the L-PA group did not differ in their baseline performance but only the L-PA group showed a significant effect on phonological fluency.

To our knowledge, this is the first study documenting facilitation of a linguistic task by prismatic adaptation, i.e. a procedure traditionally associated with modulation of spatial cognition or cognitive functions linked to spatial components.

According to recent findings, suggesting that prismatic adaptation increases excitability of frontal and parietal areas ipsilateral to the deviation side ${ }^{11,20,21}$, we may interpret the present results as reflecting a boosting of brain excitability of left hemispheric brain regions that are also associated with phonological fluency tasks. In this field, neuroimaging and neuropsychological studies show that phonological fluency recruits a left lateralized network including inferior frontal gyrus, motor cortices, anterior cingulate, temporal regions, superior parietal cortex, hippocampus, thalamus and cerebellum $27,28,29,30,31,32$. All these areas are part of a dorsal language network ${ }^{33}$ encompassing the left fronto-temporal arcuate fasciculus ${ }^{34}$, a finding consistent with the articulatory component of the phonological fluency tasks. On the other hand, the motor articulatory component in linguistic tasks is associated with recruitment of motor cortical circuits ${ }^{35}$. The increase in the number of syllables produced for each word is also consistent with the recruitment of frontal motor areas ${ }^{36}$.

The literature shows modulation of other brain regions, in addition to frontal ones, by prismatic adaptation. Neuroimaging and neurophysiological studies support the idea that PA affects the visual attention and sensorimotor networks, including the parietal cortex and the cerebellum $37,38,39,40$. The activation of the parietal cortex and the cerebellum has been related to error collection and realignment during prismatic adaptation. The anterior cingulate cortex is also activated in an early error-correcting 
phase $^{39}$. Interestingly, parietal cortex and cerebellum are also activated during phonological fluency tasks $^{41,42}$.

It is therefore possible that phonological fluency modulation is also controlled by the parieto-cerebellar network, activated during the spatial realignment.

The grammatical class effect encountered in phonological boosting following leftward PA, with greater production of nouns than verbs, could depend on different factors. A neuroanatomical account posits that verb processing is mainly supported by the left frontal cortex while noun processing is supported by left temporal regions $43,44,45,46$. On the other hand, other evidence suggests that left frontal, parietal and temporal areas are similarly correlated with the noun and verb processing $47,48,49,50,51,52,53$. Since PA modulates a network encompassing both frontal and parieto-temporal areas, the grammatical class effect encountered in the present study could reflect linguistic rather than strictly anatomical factors. In particular, it has been reported that verbs are semantically more complex, they have a lower imageability and less perceptual features than nouns ${ }^{54,55,56}$. Also, verbs would be morphologically more complex ${ }^{57,58}$. These factors could partly explain greater facilitation of nouns production following modulation of a left hemispheric network by left PA. Moreover, while PA increases beta power in motor cortices ipsilateral to prismatic deviation ${ }^{21}$, verb retrieval is associated with beta suppression in motor areas ${ }^{59}$.

Modulation of a phonological fluency task by leftward prismatic adaptation fits the general idea that cognition is grounded on sensorimotor interactions. According to this, the significant changes of brain activation in regions related to sensorimotor learning following PA have been correlated to prism aftereffects beyond sensorimotor learning and extending to higher cognitive functions.

A recent rTMS study ${ }^{23}$ showed that low-frequency rTMS of the right inferior frontal gyrus increased subjects' performance in phonological fluency tasks. The results were interpreted as reflecting plastic neural changes in the left lateral frontal cortex induced by low-frequency rTMS, suppressing interhemispheric inhibitory transcallosal interactions. Interestingly, an electrophysiological study reported that leftward PA increases transcallosal interhemispheric inhibition from the left to the right primary motor cortex ${ }^{60}$. The results of the present study may, therefore, be associated to both an increase of the left frontal excitability and modulation of transcallosal inhibition, with a reduction of activity of homologous regions of the right hemisphere, as in the reported rTMS study ${ }^{23}$.

Previous findings reported that rightward prismatic adaptation does not produce significant cognitive changes in healthy subjects ${ }^{61,62,63,64,65,66,67,7,68}$, (but see ${ }^{69,21}$ for neurophysiological changes of brain activities in healthy adults). The authors interpreted this asymmetry of prismatic adaptation effects as related to the right hemisphere dominance in visual attention networks ${ }^{70,71}$. This dominance would explain the phenomenon of leftward attentional bias called pseudoneglect. Indeed, leftward PA can counteract pseudoneglect, while rightward PA would be less efficient in shifting attention further towards the left hemispace. Therefore, one may think that the selective effects of leftward optical deviation on phonological fluency could also be linked to the modulation of spatial factors selectively in this 
condition. Indeed, the significant correlation between spatial aftereffect and phonological fluency is in line with this hypothesis.

The influence of spatial components on linguistic representations has been reported in the literature. Turriziani et al. ${ }^{72}$ described attentional representational biases in semantic judgments in healthy subjects, similar to those observed for the processing of space and numbers. Spatial manipulation of semantics was linked to the activation of specialized attentional resources located in the left hemisphere, and it was selectively modulated by left parietal rTMS. One could argue that there could be an influence of spatial factors also in the phonological fluency task. This task requires to produce as many words as possible in a restricted time based on the predefined criterion. A leftward spatial bias has been reported for mental representations of alphabet lines. This bias is counteracted by leftward but not rightward $\mathrm{PA}^{73}$. Therefore, assuming that the representation of alphabet letters could be spatially organized in a left-to-right pattern, it could be hypothesized that in the present study leftward PA has shifted attention to the right space and facilitated focusing of attention to the ending letter targets (i.e. "S"). Although the hypothesis is intriguing for future, at present it only remains speculative and further, dedicated, studies, will be necessary to test this prediction.

If confirmed and extended to clinical populations of neurological patients, the present findings could help to devise a novel type of non-invasive neuromodulation approaches for cortical dysfunctions involving the left hemisphere. In this field, since fluency tasks lie at the interface between language and executive functions, and can be impaired in numerous neurological disorders, their neuromodulation could have a huge clinical impact for a variety of disorders.

\section{References}

1. Redding, G. M. \& Wallace, B. Prism adaptation and unilateral neglect: Review and analysis. Neuropsychologia (2006). doi:10.1016/j.neuropsychologia.2005.04.009

2. Michel, C. Beyond the sensorimotor plasticity: Cognitive Expansion of Prism Adaptation in Healthy Individuals. Frontiers in Psychology (2016). doi:10.3389/fpsyg.2015.01979

3. Rossetti, Y., Kitazawa, S. \& Nijboer, T. Prism adaptation: From rehabilitation to neural bases. Cortex (2019). doi:10.1016/j.cortex.2019.01.002

4. Jewell, G. \& McCourt, M. E. Pseudoneglect: A review and meta-analysis of performance factors in line bisection tasks. Neuropsychologia (2000). doi:10.1016/S0028-3932(99)00045-7

5. Oliveri, M. et al. Overestimation of numerical distances in the left side of space. Neurology (2004). doi:10.1212/01.WNL.0000145975.58478.6D

6. Vangkilde, S. \& Habekost, T. Finding Wally: Prism adaptation improves visual search in chronic neglect. Neuropsychologia (2010). doi:10.1016/j.neuropsychologia.2010.03.020

7. Striemer, C. L. \& Danckert, J. A. Through a prism darkly: Re-evaluating prisms and neglect. Trends Cogn. Sci. (2010). doi:10.1016/j.tics.2010.04.001 
8. Magnani, B., Oliveri, M., Renata Mangano, G. \& Frassinetti, F. The role of posterior parietal cortex in spatial representation of time: A TMS study. Behav. Neurol. (2010). doi:10.3233/BEN-2010-0298

9. Magnani, B., Oliveri, M., Mancuso, G., Galante, E. \& Frassinetti, F. Time and spatial attention: Effects of prism adaptation on temporal deficits in brain damaged patients. Neuropsychologia (2011). doi:10.1016/j.neuropsychologia.2010.12.014

10. Magnani, B., Mangano, G. R., Frassinetti, F. \& Oliveri, M. The role of posterior parietal cortices on prismatic adaptation effects on the representation of time intervals. Neuropsychologia (2013). doi:10.1016/j.neuropsychologia.2013.08.006

11. Magnani, B. et al. Left insular cortex and left SFG underlie prismatic adaptation effects on time perception: Evidence from fMRI. Neuroimage (2014). doi:10.1016/j.neuroimage.2014.01.028

12. Oliveri, M., Magnani, B., Filipelli, A., Avanzi, S. \& Frassinetti, F. Prismatic adaptation effects on spatial representation of time in neglect patients. Cortex (2013). doi:10.1016/j.cortex.2011.11.010

13. Frassinetti, F., Magnani, B. \& Oliveri, M. Prismatic lenses shift time perception. Psychol. Sci. (2009). doi:10.1111/j.1467-9280.2009.02390.x

14. Anelli, F. \& Frassinetti, F. Prisms for timing better: A review on application of prism adaptation on temporal domain. Cortex (2019). doi:10.1016/j.cortex.2018.10.017

15. Striemer, C. L. \& Danckert, J. Dissociating perceptual and motor effects of prism adaptation in neglect. Neuroreport (2010). doi:10.1097/WNR.0b013e328338592f

16. Michel, C., Bonnet, C., Podor, B., Bard, P. \& Poulin-Charronnat, B. Wearing prisms to hear differently: After-effects of prism adaptation on auditory perception. Cortex (2019). doi:10.1016/j.cortex.2019.01.015

17. Christophe, L. et al. Prisms to Shift Pain Away: Pathophysiological and Therapeutic Exploration of CRPS with Prism Adaptation. Neural Plast. (2016). doi:10.1155/2016/1694256

18. Rode, G., Klos, T., Courtois-Jacquin, S., Rossetti, Y. \& Pisella, L. Neglect and prism adaptation: A new therapeutic tool for spatial cognition disorders. Restorative Neurology and Neuroscience (2006).

19. Schintu, S., Freedberg, M., Alam, Z. M., Shomstein, S. \& Wassermann, E. M. Left-shifting prism adaptation boosts reward-based learning. Cortex (2018). doi:10.1016/j.cortex.2018.09.021

20. Bracco, M., Mangano, G. R., Turriziani, P., Smirni, D. \& Oliveri, M. Combining tDCS with prismatic adaptation for non-invasive neuromodulation of the motor cortex. Neuropsychologia (2017). doi:10.1016/j.neuropsychologia.2017.05.006

21. Bracco, M., Veniero, D., Oliveri, M. \& Thut, G. Prismatic adaptation modulates oscillatory EEG correlates of motor preparation but not visual attention in healthy participants. J. Neurosci. (2018). doi:10.1523/JNEUROSCI.1422-17.2017

22. Gutierrez-Sigut, E., Payne, H. \& MacSweeney, M. Investigating language lateralization during phonological and semantic fluency tasks using functional transcranial Doppler sonography. Laterality (2015). doi:10.1080/1357650X.2014.914950 
23. Smirni, D. et al. Modulating phonemic fluency performance in healthy subjects with transcranial magnetic stimulation over the left or right lateral frontal cortex. Neuropsychologia (2017). doi:10.1016/j.neuropsychologia.2017.06.006

24. Baldo, J. V., Schwartz, S., Wilkins, D. P. \& Dronkers, N. F. Double dissociation of letter and category fluency following left frontal and temporal lobe lesions. Aphasiology (2010). doi:10.1080/02687038.2010.489260

25. Metternich, B., Buschmann, F., Wagner, K., Schulze-Bonhage, A. \& Kriston, L. Verbal fluency in focal epilepsy: A systematic review and meta-analysis. Neuropsychology Review (2014). doi:10.1007/s11065-014-9255-8

26. Rodríguez-Aranda, C. et al. Neuroanatomical correlates of verbal fluency in early Alzheimer's disease and normal aging. Brain Lang. (2016). doi:10.1016/j.bandl.2016.03.001

27. Phelps, E. A., Hyder, F., Blamire, A. M. \& Shulman, R. G. FMRI of the prefrontal cortex during overt verbal fluency. Neuroreport (1997). doi:10.1097/00001756-199701200-00036

28. Gourovitch, M. L. et al. A comparison of rCBF patterns during letter and semantic fluency. Neuropsychology (2000). doi:10.1037/0894-4105.14.3.353

29. Abrahams, S. et al. Functional magnetic resonance imaging of verbal fluency and confrontation naming using compressed image acquisition to permit overt responses. Hum. Brain Mapp. (2003). doi:10.1002/hbm.10126

30. Costafreda, S. G. et al. A systematic review and quantitative appraisal of fMRI studies of verbal fluency: Role of the left inferior frontal gyrus. Human Brain Mapping (2006). doi:10.1002/hbm.20221

31. Robinson, G., Shallice, T., Bozzali, M. \& Cipolotti, L. The differing roles of the frontal cortex in fluency tests. Brain (2012). doi:10.1093/brain/aws142

32. Biesbroek, J. M. et al. Shared and distinct anatomical correlates of semantic and phonemic fluency revealed by lesion-symptom mapping in patients with ischemic stroke. Brain Struct. Funct. (2016). doi:10.1007/s00429-015-1033-8

33. Hickok, G. \& Poeppel, D. The cortical organization of speech processing. Nature Reviews Neuroscience (2007). doi:10.1038/nrn2113

34. Blecher, T., Miron, S., Schneider, G. G., Achiron, A. \& Ben-Shachar, M. Association between white matter microstructure and verbal fluency in patients with multiple sclerosis. Front. Psychol. (2019). doi:10.3389/fpsyg.2019.01607

35. Oliveri, M. et al. All talk and no action: A transcranial magnetic stimulation study of motor cortex activation during action word production. J. Cogn. Neurosci. (2004).

doi:10.1162/089892904322926719

36. Wildgruber, D., Ackermann, H. \& Grodd, W. Differential contributions of motor cortex, basal ganglia, and cerebellum to speech motor control: Effects of syllable repetition rate evaluated by fMRI. Neuroimage (2001). doi:10.1006/nimg.2000.0672

37. Chapman, H. L. et al. Neural mechanisms underlying spatial realignment during adaptation to optical wedge prisms. Neuropsychologia (2010). doi:10.1016/j.neuropsychologia.2010.05.006 
38. Clower, D. M. et al. Role of posterior parietal cortex in the recalibration of visually guided reaching. Nature (1996). doi:10.1038/383618a0

39. Danckert, J., Ferber, S. \& Goodale, M. A. Direct effects of prismatic lenses on visuomotor control: An event-related functional MRI study. Eur. J. Neurosci. (2008). doi:10.1111/j.1460-9568.2008.06460.x

40. Luauté, J. et al. Dynamic changes in brain activity during prism adaptation. J. Neurosci. (2009). doi:10.1523/JNEUROSCI.3054-08.2009

41. Ben-Yehudah, G. \& Fiez, J. A. Impact of cerebellar lesions on reading and phonological processing. in Annals of the New York Academy of Sciences (2008). doi:10.1196/annals.1416.015

42. Mariën, P. et al. Consensus paper: Language and the cerebellum: An ongoing enigma. Cerebellum (2014). doi:10.1007/s12311-013-0540-5

43. Damasio, A. R. \& Tranel, D. Nouns and verbs are retrieved with differently distributed neural systems. in Proceedings of the National Academy of Sciences of the United States of America (1993). doi:10.1073/pnas.90.11.4957

44. Daniele, A., Giustolisi, L., Silveri, M. C., Colosimo, C. \& Gainotti, G. Evidence for a possible neuroanatomical basis for lexical processing of nouns and verbs. Neuropsychologia (1994). doi:10.1016/0028-3932(94)00066-2

45. Shapiro, K. A., Moo, L. R. \& Caramazza, A. Cortical signatures of noun and verb production. Proc. Natl. Acad. Sci. U. S. A. (2006). doi:10.1073/pnas.0504142103

46. Cappelletti, M., Fregni, F., Shapiro, K., Pascual-Leone, A. \& Caramazza, A. Processing nouns and verbs in the left frontal cortex: A transcranial magnetic stimulation study. J. Cogn. Neurosci. (2008). doi:10.1162/jocn.2008.20045

47. Aggujaro, S., Crepaldi, D., Pistarini, C., Taricco, M. \& Luzzatti, C. Neuro-anatomical correlates of impaired retrieval of verbs and nouns: Interaction of grammatical class, imageability and actionality. J. Neurolinguistics (2006). doi:10.1016/j.jneuroling.2005.07.004

48. Kemmerer, D., Rudrauf, D., Manzel, K. \& Tranel, D. Behavioral patterns and lesion sites associated with impaired processing of lexical and conceptual knowledge of actions. Cortex (2012). doi:10.1016/j.cortex.2010.11.001

49. Luzzatti, C., Aggujaro, S. \& Crepaldi, D. Verb-noun double dissociation in aphasia: Theoretical and neuroanatomical foundations. Cortex (2006). doi:10.1016/S0010-9452(08)70431-3

50. Tranel, D., Adolphs, R., Damasio, H. \& Damasio, A. R. A neural basis for the retrieval of words for actions. Cogn. Neuropsychol. (2001). doi:10.1080/02643290126377

51. Li, P., Jin, Z. \& Tan, L. H. Neural representations of nouns and verbs in Chinese: An fMRI study. Neuroimage (2004). doi:10.1016/j.neuroimage.2003.10.044

52. Siri, S. et al. The neural substrate of naming events: Effects of processing demands but not of grammatical class. Cereb. Cortex (2008). doi:10.1093/cercor/bhm043

53. Crepaldi, D., Berlingeri, M., Paulesu, E. \& Luzzatti, C. A place for nouns and a place for verbs? A critical review of neurocognitive data on grammatical-class effects. Brain and Language (2011). 
doi:10.1016/j.bandl.2010.09.005

54. Bird, H., Howard, D. \& Franklin, S. Why is a verb like an inanimate object? Grammatical category and semantic category deficits. Brain Lang. (2000). doi:10.1006/brln.2000.2292

55. Breedin, S. D., Saffran, E. M. \& Schwartz, M. F. Semantic factors in verb retrieval: An effect of complexity. Brain Lang. (1998). doi:10.1006/brln.1997.1923

56. McCarthy, R. \& Warrington, E. K. Category specificity in an agrammatic patient: The relative impairment of verb retrieval and comprehension. Neuropsychologia (1985). doi:10.1016/00283932(85)90079-X

57. Badecker, W. \& Caramazza, A. Morphological composition in the lexical output system. Cogn. Neuropsychol. (1991). doi:10.1080/02643299108253377

58. Tsapkini, K., Jarema, G. \& Kehayia, E. A morphological processing deficit in verbs but not in nouns: A case study in a highly inflected language. J. Neurolinguistics (2002). doi:10.1016/S09116044(01)00039-2

59. Pavlova, A. A. et al. Effortful verb retrieval from semantic memory drives beta suppression in mesial frontal regions involved in action initiation. Hum. Brain Mapp. (2019). doi:10.1002/hbm.24624

60. Martín-Arévalo, E., Schintu, S., Farnè, A., Pisella, L. \& Reilly, K. T. Adaptation to Leftward Shifting Prisms Alters Motor Interhemispheric Inhibition. Cereb. Cortex (2018). doi:10.1093/cercor/bhw386

61. Berberovic, N. \& Mattingley, J. B. Effects of prismatic adaptation on judgements of spatial extent in peripersonal and extrapersonal space. Neuropsychologia (2003). doi:10.1016/S00283932(02)00090-8

62. Goedert, K. M., Leblanc, A., Tsai, S. W. \& Barrett, A. M. Asymmetrical effects of adaptation to left- and right-Shifting prisms depends on pPre-existing attentional biases. J. Int. Neuropsychol. Soc. (2010). doi:10.1017/S1355617710000597

63. Schintu, S. et al. The asymmetrical effect of leftward and rightward prisms on intact visuospatial cognition. Cortex (2017). doi:10.1016/j.cortex.2017.09.015

64. Colent, C., Pisella, L., Bernieri, C., Rode, G. \& Rossetti, Y. Cognitive bias induced by visuo-motor adaptation to prisms: A simulation of unilateral neglect in normal individuals? Neuroreport (2000). doi:10.1097/00001756-200006260-00019

65. Michel, C., Rossetti, Y., Rode, G. \& Tilikete, C. After-effects of visuo-manual adaptation to prisms on body posture in normal subjects. Exp. Brain Res. (2003). doi:10.1007/s00221-002-1294-3

66. Fortis, P., Goedert, K. M. \& Barrett, A. M. Prism adaptation differently affects motor-intentional and perceptual-attentional biases in healthy individuals. Neuropsychologia (2011). doi:10.1016/j.neuropsychologia.2011.05.020

67. Nijboer, T., Vree, A., Dijkerman, C. \& Van Der Stigchel, S. Prism adaptation influences perception but not attention: Evidence from antisaccades. Neuroreport (2010). doi:10.1097/WNR.0b013e328337f95f 
68. Schintu, S. et al. Prism adaptation in the healthy brain: The shift in line bisection judgments is long lasting and fluctuates. Neuropsychologia (2014). doi:10.1016/j.neuropsychologia.2013.11.013

69. Crottaz-Herbette, S., Fornari, E. \& Clarke, S. Prismatic adaptation changes visuospatial representation in the inferior parietal lobule. J. Neurosci. (2014). doi:10.1523/JNEUROSCI.3184-13.2014

70. De Schotten, M. T. et al. A lateralized brain network for visuospatial attention. Nat. Neurosci. (2011). doi:10.1038/nn.2905

71. Kucyi, A., Hodaie, M. \& Davis, K. D. Lateralization in intrinsic functional connectivity of the temporoparietal junction with salience- and attention-related brain networks. J. Neurophysiol. (2012). doi:10.1152/jn.00674.2012

72. Turriziani, P. et al. Exploring the relationship between semantics and space. PLoS One (2009). doi:10.1371/journal.pone.0005319

73. Nicholls, M. E. R., Kamer, A. \& Loftus, A. M. Pseudoneglect for mental alphabet lines is affected by prismatic adaptation. Exp. Brain Res. (2008). doi:10.1007/s00221-008-1502-x

74. Oldfield, R. C. The assessment and analysis of handedness: The Edinburgh inventory. Neuropsychologia (1971). doi:10.1016/0028-3932(71)90067-4

75. Monaco, M., Costa, A., Caltagirone, C. \& Carlesimo, G. A. Forward and backward span for verbal and visuo-spatial data: Standardization and normative data from an Italian adult population. Neurol. Sci. (2013). doi:10.1007/s10072-012-1130-x

76. Nocentini, U., Giordano, A., Di Vincenzo, S., Panella, M. \& Pasqualetti, P. The symbol digit modalities test - Oral version: Italian normative data. Funct. Neurol. (2006).

77. Cattelani, R., Dal Sasso, F., Corsini, D. \& Posteraro, L. The Modified Five-Point Test: Normative data for a sample of Italian healthy adults aged 16-60. Neurol. Sci. (2011). doi:10.1007/s10072-011-0489-4

78. Caffarra, P., Vezzadini, G., Dieci, F., Zonato, F. \& Venneri, A. Una versione abbreviata del test di Stroop: Dati normativi nella popolazione Italiana. Nuova Riv. di Neurol. (2002).

79. Raven, J. C. Revised manual for Raven's Progressive Matrices and Vocabulary Scale. Revised manual for Raven's Progressive Matrices and Vocabulary Scale (1982).

80. Carlesimo, G. A. et al. The mental deterioration battery: Normative data, diagnostic reliability and qualitative analyses of cognitive impairment. Eur. Neurol. (1996). doi:10.1159/000117297

81. Novelli, G. et al. Tre test clinici di ricerca e produzione lessicale. Taratura su soggetti normali. Arch. Psicol. Neurol. Psichiatr. (1986).

82. Bonaventura, R. E. et al. Investigating prismatic adaptation effects in handgrip strength and in plantar pressure in healthy subjects. Gait Posture (2020). doi:10.1016/j.gaitpost.2019.12.022

\section{Tables}

Table 1. Demographic and neuropsychological data of participants. 


\begin{tabular}{|lll|}
\hline & L-PA & R-PA \\
\hline & mean (sd) & mean (sd) \\
\hline Age (yrs) & $23.43(1.86)$ & $23.53(2.79)$ \\
\hline Education (yrs) & $16.75(1.43)$ & $16.73(1.62)$ \\
\hline Handedness & $61.81(17.45)$ & $67.93(15.12)$ \\
\hline Digit Forward & $6.0(0,0)$ & $5.85(0,37)$ \\
\hline Digit Backward & $6.0(0.8)$ & $7.4(0.7)$ \\
\hline SDMT & $53.37(12.04)$ & $51.00(7.32)$ \\
\hline MFPT & $36.62(8.04)$ & $33.26(11.97)$ \\
\hline Stroop Test (sec) & $11.01(9.68)$ & $19.21(23.58)$ \\
\hline RAPM & $19.31(6.09)$ & $17.53(5.62)$ \\
\hline
\end{tabular}

Figures 

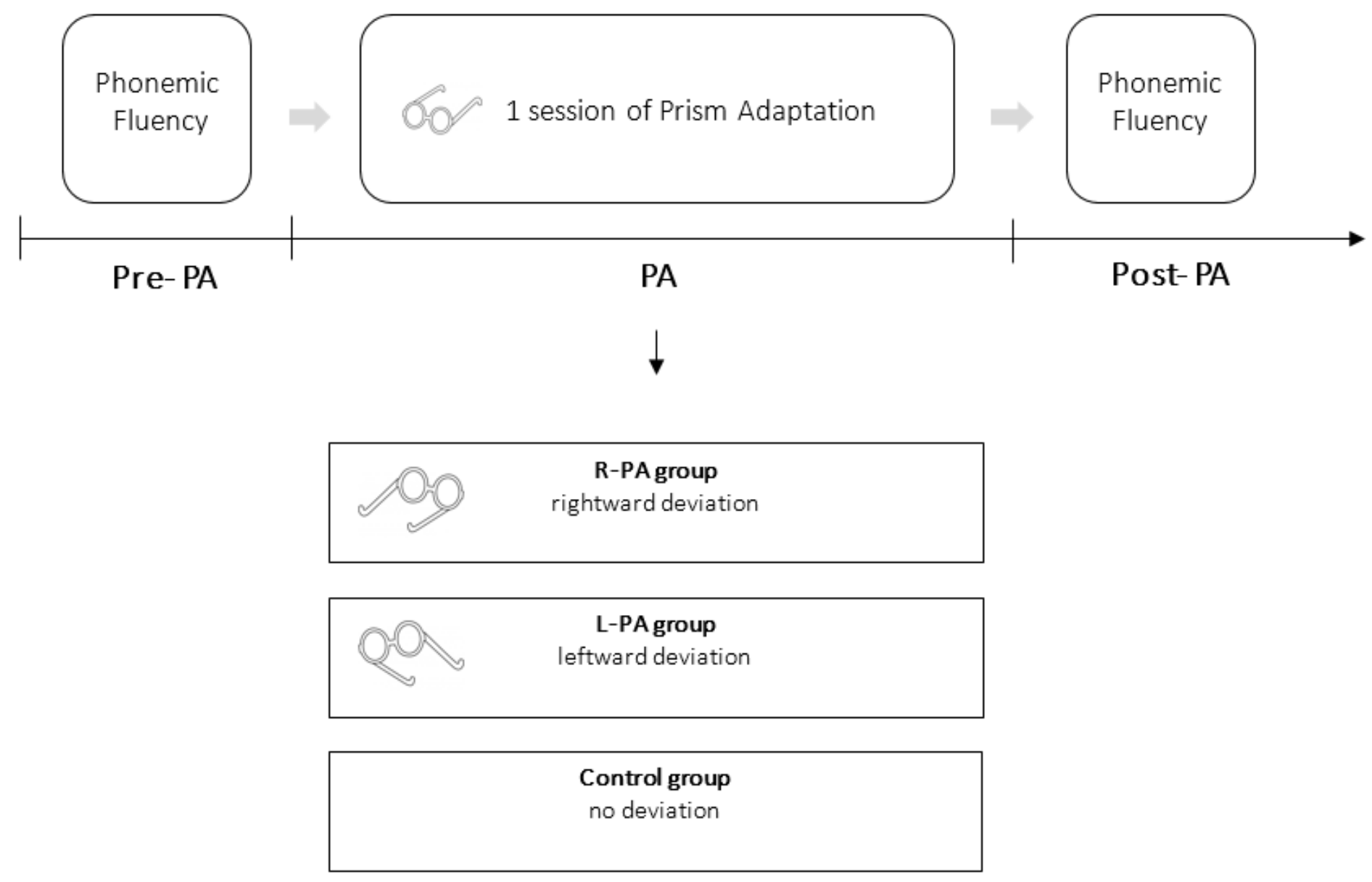

\section{Figure 1}

Schematic representation of the experimental design.

A
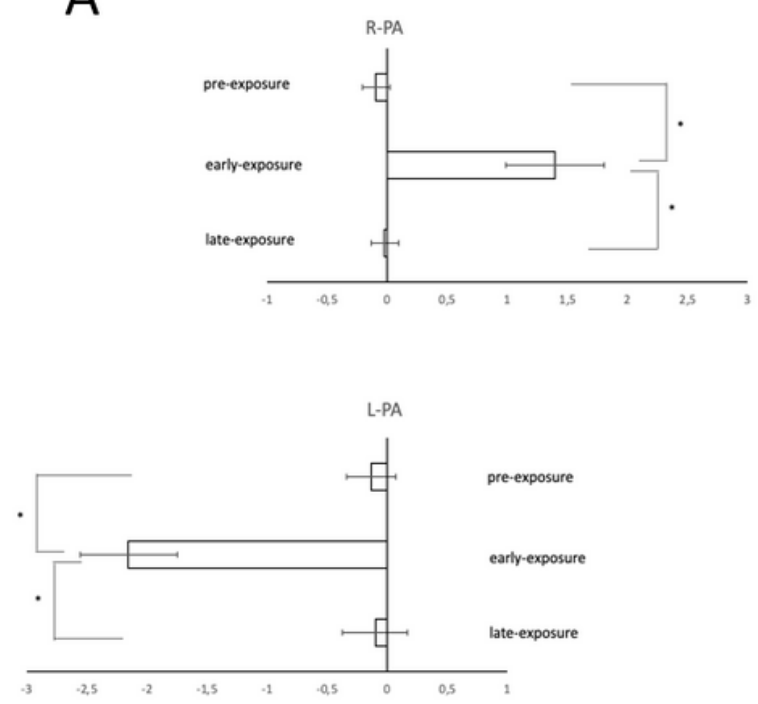
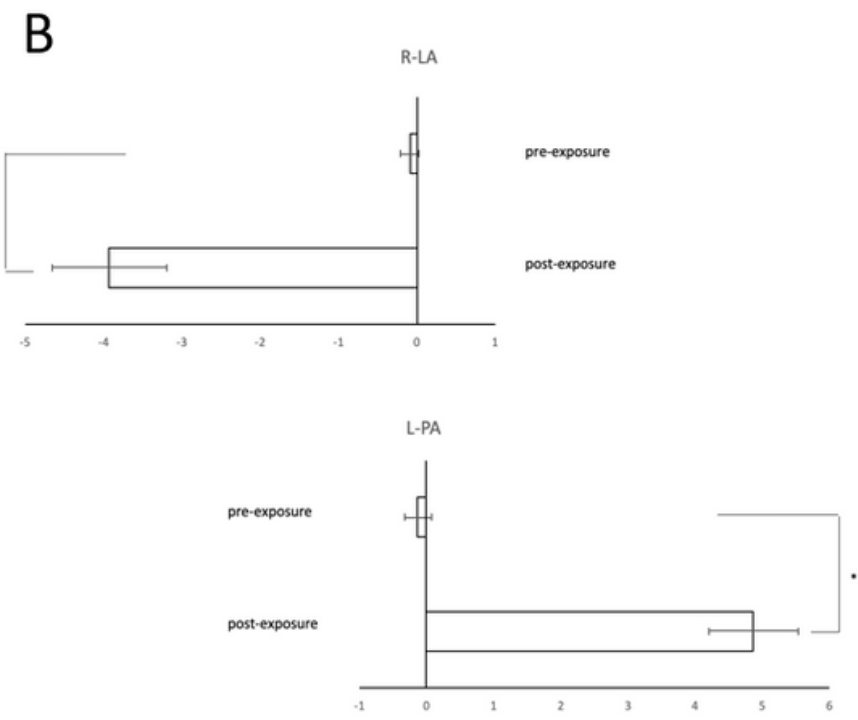
Figure 2

Prismatic adaptation parameters: A) error reduction and B) aftereffect. Mean pointing displacement in four experimental conditions across groups (leftward prismatic adaptation group and rightward prismatic adaptation group). Legend: I-PA=leftward prismatic adaptation group; r-PA=rightward prismatic adaptation group; Error bars=standard error of mean; ${ }^{*} p<.05$. Negative values indicate leftward pointing displacement, positive values indicate rightward pointing displacement.

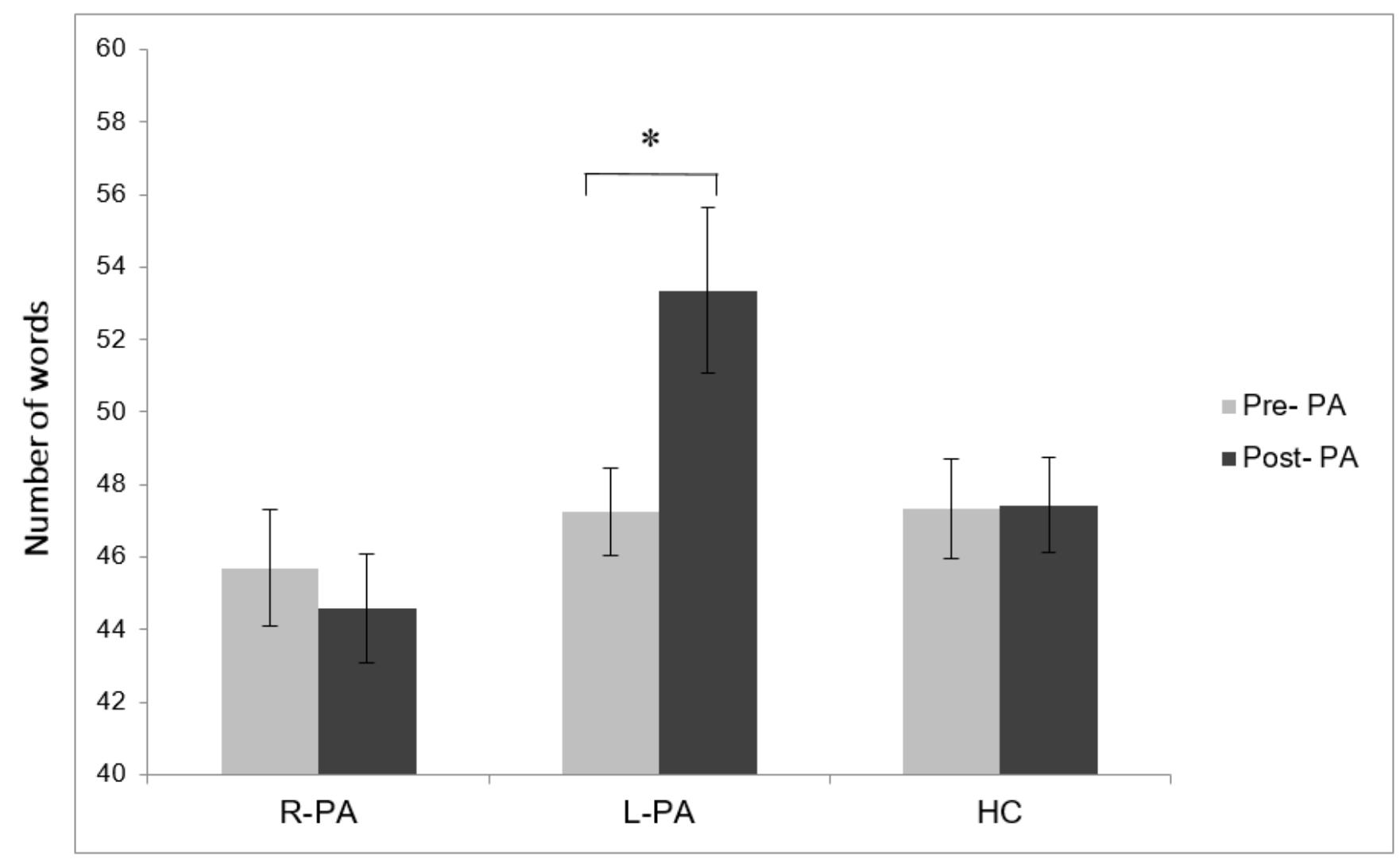

\section{Figure 3}

Phonemic fluency performance before and after prismatic adaptation (pre- PA, post-PA) across groups (LPA group, R-PA group and no PA group). L-PA significantly improves the performance on Phonemic fluency task. 